\title{
LGALS3BP: A Potential Plasma Biomarker Associated with Diagnosis and Prognosis in Patients with Sepsis
}

\author{
Meiyan Luo ${ }^{1-3}$ \\ Qian Zhang ${ }^{1-3}$ \\ Yingchun $\mathrm{Hu}^{4}$ \\ Changfeng Sun ${ }^{1-3}$ \\ Yunjian Sheng ${ }^{I-3}$ \\ Cunliang Deng ${ }^{1-3}$
}

'Department of Infectious Diseases, The Affiliated Hospital of Southwest Medical University, Louzhou, 646000, People's

Republic of China; ${ }^{2}$ Department of Tuberculosis, The Affiliated Hospital of Southwest Medical University, Louzhou, 646000 , People's Republic of China; ${ }^{3}$ Infection and Immunity Laboratory, The Affiliated Hospital of Southwest Medical University, Louzhou, 646000, People's Republic of China; ${ }^{4}$ Department of Emergency, The Affiliated Hospital of Southwest Medical University, Louzhou, 646000, People's Republic of China
Correspondence: Cunliang Deng Department of Infectious Diseases, The Affiliated Hospital of Southwest Medical University, No. 25 Taiping Street, Louzhou, 646000, People's Republic of China

Email dengcun164@vip.sina.com
Purpose: This study aimed to screen differentially expressed proteins (DEPs) in plasma of patients with sepsis through data-independent acquisition (DIA) and enzyme-linked immunosorbent assays (ELISAs), and provide convenient and accurate serum markers for determining the condition of septic patients.

Methods: A total of 53 septic patients and 16 normal controls who were admitted to the Affiliated Hospital of Southwest Medical University between January 2019 and December 2020 were enrolled in this study; 6 specimens from the normal group and 15 from the sepsis group were randomly selected for DIA-based quantitative proteomic analysis. The acquired data were subjected to Gene Ontology (GO) and Kyoto Encyclopedia of Genes and Genomes (KEGG) analysis, and a protein-protein interaction (PPI) network was constructed to screen potential markers. The selected proteins were further verified through ELISAs. The differences between control and sepsis groups and between survivors and nonsurvivors were analysed. Receiver operating characteristic (ROC) curves were drawn to explore their diagnostic value and prognostic efficacy.

Results: A total of 149 DEPs were identified by bioinformatics methods. The analyses showed that these proteins are mainly involved in biological processes such as cell movement, stress response, cell proliferation, and immune response. Functional pathway analysis showed that they are mainly involved in leukocyte transendothelial migration, protein synthesis and processing, and various bacterial infections. LGALS3BP was selected as a potential plasma biomarker and further verified through an ELISA. Its level in septic patients was significantly higher than that in normal controls, and its level in non-survivors was also higher than that in survivors. The ROC curves suggested its great diagnostic efficacy and prognostic ability in sepsis.

Conclusion: LGALS3BP levels were significantly different between the normal and sepsis groups; it has good diagnostic value in sepsis, and is related to patient prognosis; thus, it might be a biomarker for sepsis.

Keywords: LGALS3BP, sepsis, plasma biomarker, DIA, ELISA

\section{Introduction}

Sepsis is defined as life-threatening organ dysfunction caused by the unbalanced response of the body to infection. The main pathological characteristics of patients are the increased production of inflammatory factors, highly abnormal immune function, multiple organ dysfunction and even death. ${ }^{1,2}$ According to statistics, there are approximately 20 million cases of sepsis every year worldwide. ${ }^{3}$ Moreover, the fatality rate is up to $30 \%$ due to its rapid onset. If combined with septic shock, the fatality rate can be as high as $80 \% .{ }^{4}$ There are currently no definite 
and effective methods for the treatment of sepsis. Clinical treatment is based mainly on anti-infection, the correction of acidosis, body fluid resuscitation, body fluid support, haemoperfusion therapy, and mechanical ventilation. At present, a new treatment bottleneck has been reached. Even after the use of powerful antibacterial drugs for sterilization, the "waterfall effect" produced by subsequent inflammatory factors cannot be reversed, and the patient can die within a few days. Although new diagnostic criteria for sepsis were published in $2016,{ }^{5}$ the diagnostic value and prognostic value of previous biomarkers such as procalcitonin (PCT) and lactic acid in sepsis are unclear, and new biomarkers are urgently needed.

Data-independent acquisition (DIA) based quantitative proteomics is a new technology. This technology has the advantages of good repeatability, high sensitivity, high quantitative accuracy, high throughput, and data informatization. $^{6}$ It was rated as the most noteworthy technology in 2015 by Nature Methods magazine. Because DIA mode divides the entire scanning range into several window areas, all ions in each window area are selected and fragmented at high speed and cyclically to obtain all fragment information from a sample without omission and bias. ${ }^{7}$ Through DIA technology, differentially expressed proteins (DEPs) in the serum of patients with sepsis can be screened more extensively. Enzymelinked immunosorbent assays (ELISAs) are the most widely used technique in enzyme immunoassay technology. The substrate is catalysed by enzymes to produce a colour reaction, so it can be qualitatively or quantitatively observed according to the presence or absence of colour and the depth of colour. ${ }^{8}$

Bioinformatics is now widely used to process large-scale mass spectrometry data, and can be used to comprehensively analyse changes in protein expression. It has also become an important method for the study of disease pathogenesis. Gene Ontology (GO) analysis is commonly used to study gene functions and includes three parts: biological process, cellular component and molecular function. The molecular functions that gene products may perform, the cellular environment in which they are located, and the biological processes involved are described. Kyoto Encyclopedia of Genes and Genomes (KEGG) can be used to analyse the related signalling pathways and in which these proteins participate. A protein-protein interaction (PPI) network and be constructed. Taking each protein as a node and its connection with other related proteins as edges, a complex network is formed. The corresponding software is used to describe the interconnections and interactions between various proteins in a network.

Through DIA and ELISAs, DEPs can be screened at one time, and new biomarkers with diagnostic and prognostic significance can be identified by combining patients' clinical information and provide clinicians with new diagnostic tools.

\section{Materials and Methods \\ Recruitment of Subjects and Collection of Blood}

In this study, blood samples were collected from septic patients $(n=53)$ and normal volunteers $(n=16)$ in the Department of Emergency Intensive Care Unit (EICU) of the Affiliated Hospital of Southwest Medical University from January to December 2019. The inclusion criteria were as follows: 1. Met the definition of sepsis 3.0 and the diagnostic criteria issued by the American Society of Critical Care Medicine (SCCM) and European Society of Intensive Care Medicine (ESICM); 2. Aged between 16 and 75 years; and 3. The subjects or legal representatives were willing to enter the study and sign an informed consent form. Patients were excluded if they were $<16$ years old, if they had an imminently terminal comorbid condition, or if they were participating in an ongoing clinical trial. Written informed consent was obtained from all study participants or their legal designates.

\section{DIA Quantitative Proteomic Analysis}

6 blood samples from the normal group and 15 from the sepsis group were randomly selected for DIA proteomics analysis. For this project, we used the Q-Exactive HF (Thermo Fisher Scientific, San Jose, CA) instrument in DIA mode to achieve accurate and highly repeatable quantitative mass spectrometry data on a large number of proteins. By combining the DDA database and MSstats software, we performed qualitative and quantitative information analyses on the peptides and proteins obtained from the mass spectrometry analysis to obtain a large number of reliable quantitative results.

\section{Screening Target Genes}

All data were processed logarithmically. Box diagrams and principal component analysis were used to judge the uniformity of samples and discrimination between groups. The DEPs between the normal group and the sepsis group were screened with the online tool iDEP91 (http://bioinformatics. sdstate.edu/idep/), ${ }^{9}$ and the screening criteria were fold change 
$\geq 1.5$ and false discovery rate (FDR) $<0.01$. The DEPs were submitted to the STRING (https://string-db.org) online platform, ${ }^{10}$ and the PPI network was constructed. Cytoscape 3.8.0 software was used to visualize the network. ${ }^{11}$ The differentially expressed proteins in the centre of the network between the normal group and the sepsis group were clarified, and potential serum markers were searched for in the PubMed database.

\section{KEGG and GO Analyses}

To further understand the functions of the DEPs in septic, the OmicShare (https://www.omicshare.com/) platform was used to perform GO and KEGG enrichment analyses. ${ }^{12}$ By performing enrichment analysis, we can preliminarily clarify the biological processes or signalling pathways which the DEPs may participate in.

\section{ELISA Validation}

In this study, the double antibody sandwich method of ELISA was adopted to detect the level of LGALS3BP in the specimens. Specimens were added to an LGALS3BP antibody-coated microtiter plate and then combined with a horseradish peroxidase (HRP)-labelled detection antibody to form an antibody-antigen-enzyme-labelled antibody complex. After thorough washing, we added the TMB chromogenic agent for visualization. The TMB ELISA substrate system was converted into blue under catalysis of the HRP enzyme and into yellow under the action of the acid. The depth of colour was positively correlated with the LGALS3BP content in the samples. The absorbance was measured with an enzyme-labelled instrument at a wavelength of $450 \mathrm{~nm}$, and the LGALS3BP content in the sample was calculated from the standard curve. The colour intensity is positively correlated with LGALS3BP in the sample.

\section{Receiver Operating Characteristic (ROC) Curve Analysis}

MedCalc 15.2 software was used to draw the ROC curves, and the area under the curve (AUC) was calculated to evaluate the diagnostic and prognostic ability of LGALS3BP. The AUCs of the ROC curves between the normal and sepsis groups and between survivors and nonsurvivors were greater than 0.7 ; therefore, LGALS3BP was considered to have diagnostic efficacy and prognostic ability.

\section{Statistical Analyses}

The data obtained from the ELISA and patients' clinical information were analysed with SPSS 22.0 software. Continuous variables are presented as the mean \pm standard deviation (SD) or median(interquartile range). Differences in continuous variables were analysed with the nonparametric Mann-Whitney $U$-test and unpaired Student's $t$-test. The chi-square test was used to carry out comparisons between categorical variables. The numbers of patients were compared by Fisher's exact test. The cutoff criterion was a $P$ value $<0.05$.

\section{Results}

\section{Demographics and Clinical Characteristics}

The clinical information of the recruited patients enrolled in this study is presented in Table 1. This study included 16 normal patients and 53 patients with sepsis. Among the septic patients, the causes of sepsis were pneumonia (22, $41.5 \%)$, abdominal infection $(18,34.0 \%)$, urinary tract

Table I Baseline Characteristics of the Recruited Patients

\begin{tabular}{|l|l|l|l|l|}
\hline Clinical Variable & Normal Control $(\mathbf{n = 1 6})$ & Sepsis $(\mathbf{n}=\mathbf{5 3})$ & Sepsis Survivor $(\mathbf{n}=33)$ & Sepsis Non-Survivor $(\mathbf{n}=\mathbf{I 5})$ \\
\hline Male & $10(62.5 \%)$ & $36(67.9 \%)$ & $23(69.7 \%)$ & $9(60.00 \%)$ \\
Female & $6(37.5 \%)$ & $17(32.1 \%)$ & $10(30.3 \%)$ & $6(40.00 \%)$ \\
Age (years) & $53.25 \pm 9.17$ & $55.68 \pm 14.26$ & $55.00 \pm 12.99$ & $61.27 \pm 13.02$ \\
WBC (I0^9//) & $6.51 \pm 1.86$ & $12.47 \pm 7.49^{\mathrm{a}}$ & $12.62 \pm 7.79$ & $13.07 \pm 7.10$ \\
PLT (I0^9/I) & $215.50(190.56,237.94)$ & $164.00(144.53,209.62)^{\mathrm{a}}$ & $164.00(136.52,225.16)$ & $189.00(116.95,238.25)$ \\
ALT (u/L) & $19.30(15.65,26.90)$ & $31.30(19.60,93.60)^{\mathrm{a}}$ & $74.16(22.53,117.38)$ & $33.80(14,25,41.88)^{\mathrm{b}}$ \\
Cre (umol/L) & $65.30(54.93,75.20)$ & $82.80(64.43,160.83)^{\mathrm{a}}$ & $113.82(64.58,122.83)$ & $161.11(46.20,215.10)$ \\
PCT (ng/mL) & NA & $28.64(1.68,40.83)$ & $25.84(0.73,38.83)$ & $34.80(2.85,78.77)$ \\
SOFA score & NA & $5.91 \pm 3.01$ & $5.58 \pm 3.18$ & $6.40 \pm 2.80$ \\
\hline
\end{tabular}

Notes: a Means compared with the normal group, $p<0.05$; ' $\mathrm{b}$ Means compared with the sepsis survivors, $\mathrm{p}<0.05$. 
infection $(6,11.3 \%)$, soft tissue infection $(5,9.4 \%)$, and bloodstream infection (2, 3.8\%) (Figure 1A). Over a median follow-up period of 28 days, in the sepsis group, 33 people survived, 15 died, and 5 were lost to follow-up, the causes of survivors and non-survivors were displayed in Figure 1B and C. No considerable differences in sex or age were found between the patients with sepsis and normal volunteers; these factors were also not different between the survivors and non-survivors. The white blood cell (WBC) count, platelet count, and alanine aminotransferase and serum creatinine levels differed significantly between normal controls and septic patients $(\mathrm{P}<0.05)$, but there were no considerable differences between survivors and non-survivors except for the alanine aminotransferase level. In addition, there were no significant differences in PCT levels or SOFA scores between survivors and non-survivors.

\section{Identification of DEPs Between Normal Controls and Septic Patients}

Through box plots and principal component analysis, the uniformity and discrimination of normal samples and sepsis samples in the DIA-based proteomics analysis were found to be excellent (Figure 2A and B), and no outliers were detected. The proteomic data were analysed with iDEP91 online software. A total of 149 DEPs were screened from the serum samples of patients in the normal group and the sepsis group. Compared with the normal group, there were 17 downregulated proteins and 132 upregulated genes in the septic group (Figure 2C and D).

\section{Proteins Networks and Hub Protein Analysis}

The DEPs were submitted to the STRING (https://stringdb.org) online platform to construct the PPI network (Figure $3 \mathrm{~A}$ ). The relationship between proteins is presented in the network. Through the PPI network, we found that LGALS3BP, CD59, MIF, TIMP1, MMP2, CD44, CD14, LTBP2 and other proteins were at the centre of the network module and differed significantly between the normal and sepsis groups $(P<0.05)$ (Figure 3B). After searching the PubMed database, we found no relevant research on LGALS3BP levels in the serum of patients with septic; therefore, its specific function in septic needed further verification.

\section{GO Classification and Pathway Enrichment of the DEPs}

The GO functional enrichment analysis of DEPs showed that these proteins are mainly involved in biological processes such as cell movement, the stress response, cell proliferation, the immune response, and intercellular signal transduction (Figure 4A). KEGG pathway analysis revealed that these DEPs are mainly related to signalling pathways such as leukocyte migration across the endothelium, protein synthesis and processing, the complement and coagulation cascade, and various bacterial infections (Figure 4B).

\section{Validation of LGALS3BP Through an ELISA}

As shown by the ELISA results in Figure 5A and B, the expression level of LGALS3BP in the serum of patients with sepsis was $119.8 \pm 32.7 \mathrm{ng} / \mathrm{mL}$, which was obviously
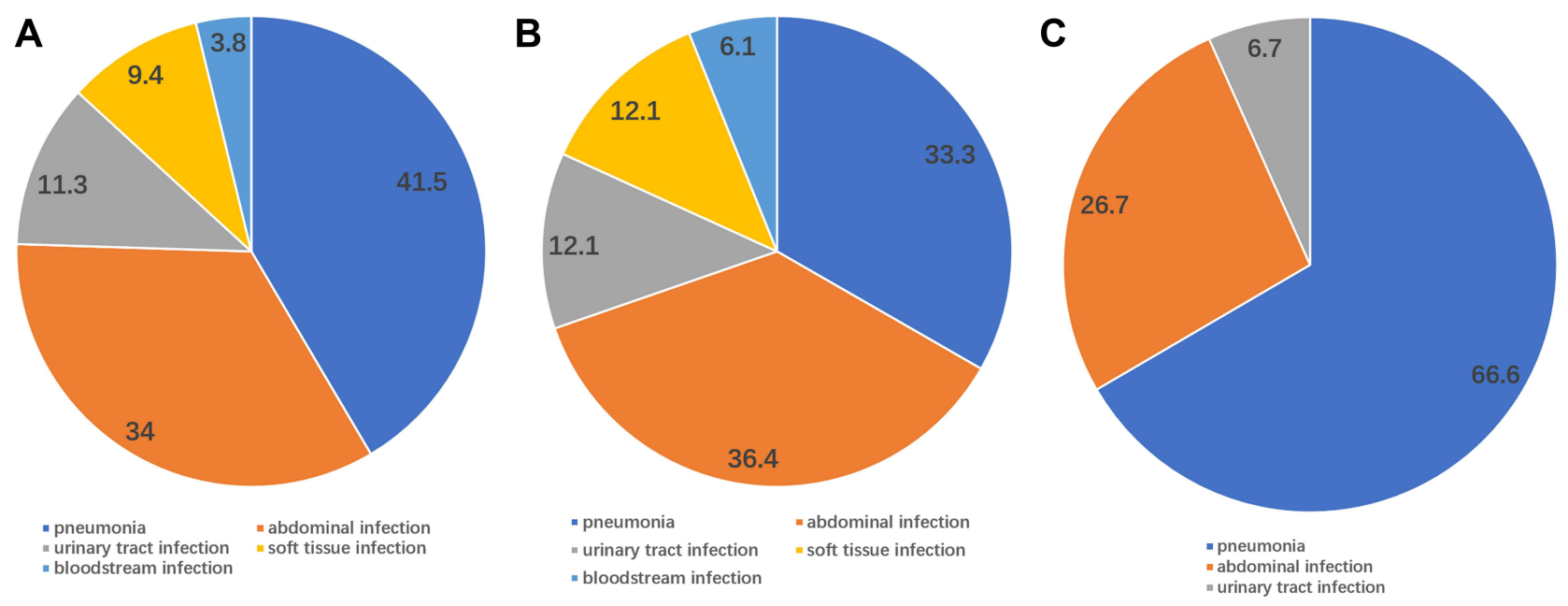

Figure I Main sources of recruited septic patients: septic patients (A), survivors (B), non-survivors (C). 

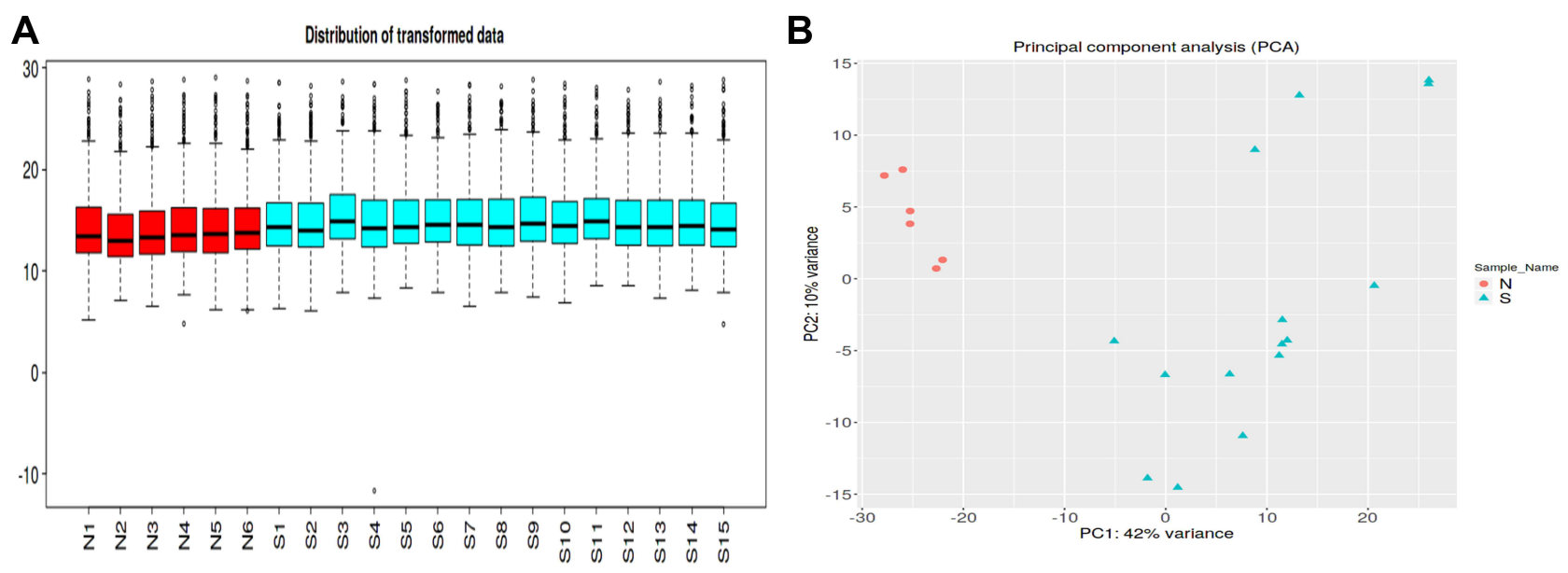

C

D
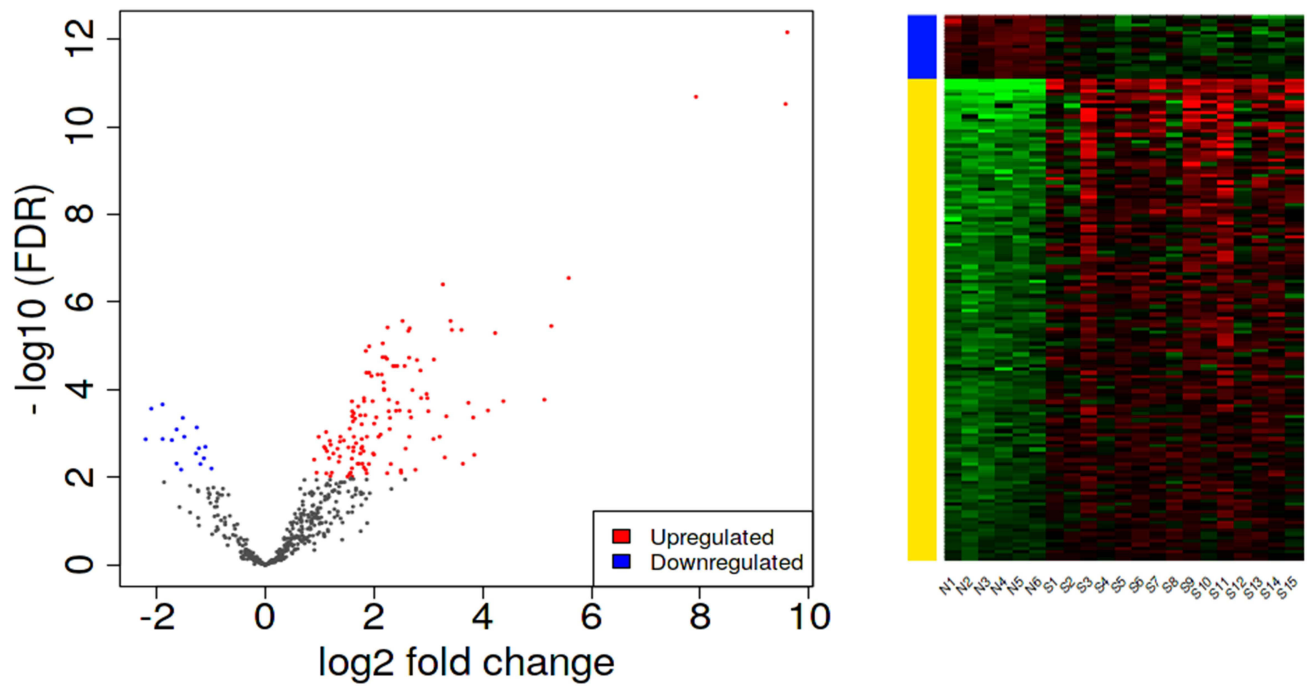

Down $(\mathrm{N}=17)$ Up ( $\mathrm{N}=132)$

Figure 2 Box plot (A), principal component analysis (B), volcano map (C), and heat map (D) of sepsis samples. The criteria for selection of differentially expressed proteins was set as fold-change $\geq 1.5$, false discovery rate $($ FDR $)<0.01$.

higher than that in normal controls $(76.1 \pm 49.2 \mathrm{ng} / \mathrm{mL})$ $(P<0.01)$ (Figure 5A). In addition, its expression level in non-survivors $(142.2 \pm 27.2 \mathrm{ng} / \mathrm{mL})$ was higher than that in survivors $(110.2 \pm 30.4 \mathrm{ng} / \mathrm{mL}$ ) (Figure 5B). Therefore, it was concluded that the expression level of LGALS3BP increased with the progression of sepsis.

\section{ROC Curve Analysis}

The ROC curves were generated based on the ELISA results. According to the results, LGALS3BP had great diagnostic efficacy for sepsis; its area under the ROC curve was 0.74 , with a sensitivity of $98.0 \%$, and a specificity of $53.3 \%$ (Figure 6A). The area under the ROC curve between survivors and non-survivors was 0.80 (Figure 6B), which indicates that LGALS3BP has prognostic ability for patients with sepsis.

\section{Discussion}

Sepsis is a disease that poses an enormous threat to a patient's health. After the illness, patients subsequently develop symptoms such as fever, tachycardia, and shortness of breath. Moreover, shock or systemic multiple organ dysfunction may gradually appear after the disease progresses. The early identification of patients at a high risk of death and effective treatment can reduce the mortality rate. In this study, through bioinformatics methods and DIA analysis, we determined that LGALS3BP has potential as a serum marker of sepsis. In combination with the ELISA results, we found that the expression level of LGALS3BP gradually increased as the disease progressed. The ROC curve revealed that LGALS3BP has diagnostic ability in sepsis, and by comparing 

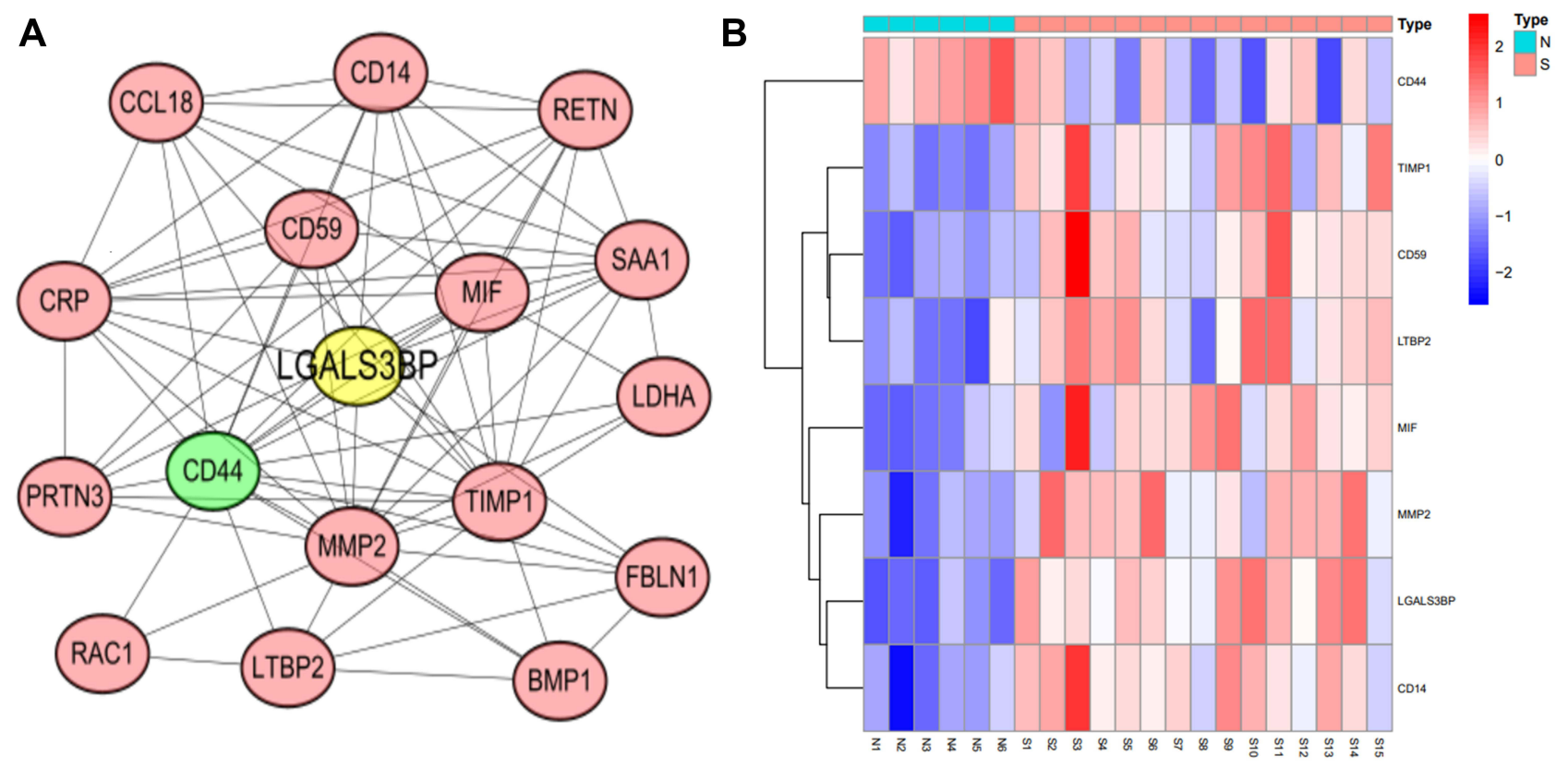

Figure 3 Protein-protein interaction network of differentially expressed proteins $(\mathbf{A})$ and heat map of proteins that were at the centre of this network (B) .

A

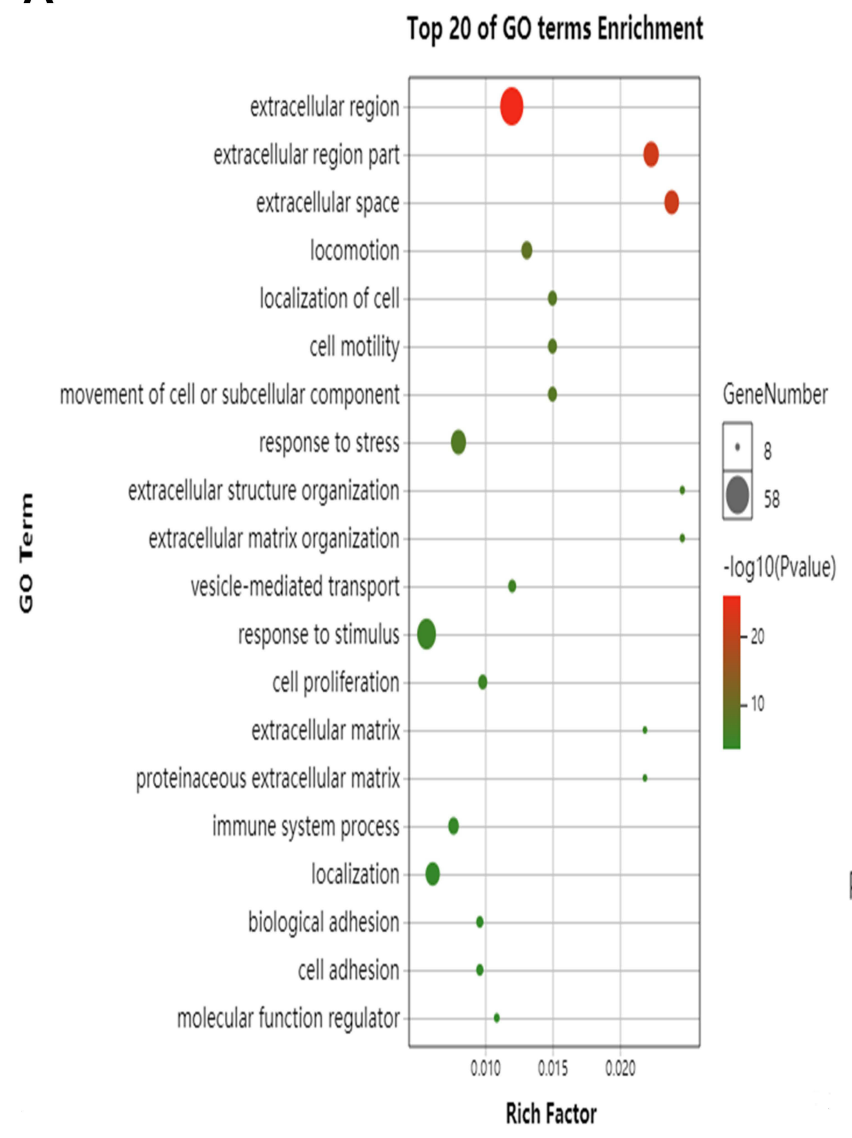

B

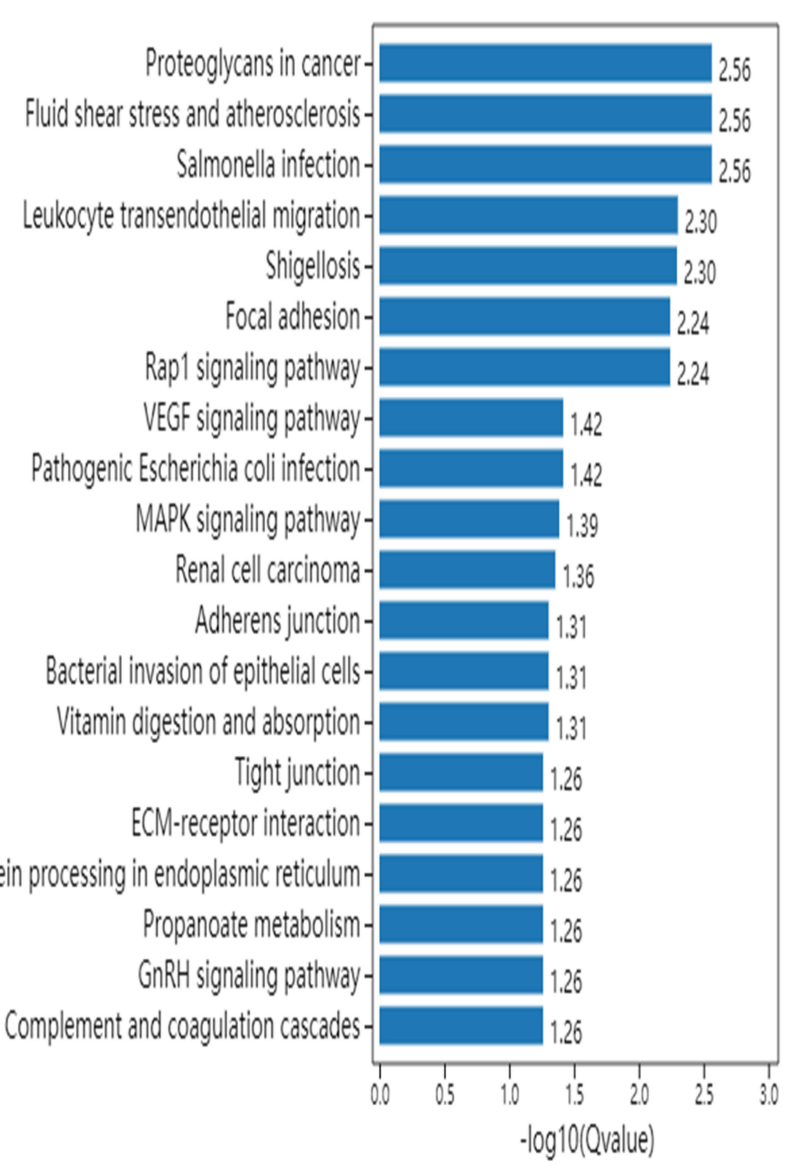

Figure 4 The results of Gene Ontology analysis (A) and KEGG pathway analysis (B). 


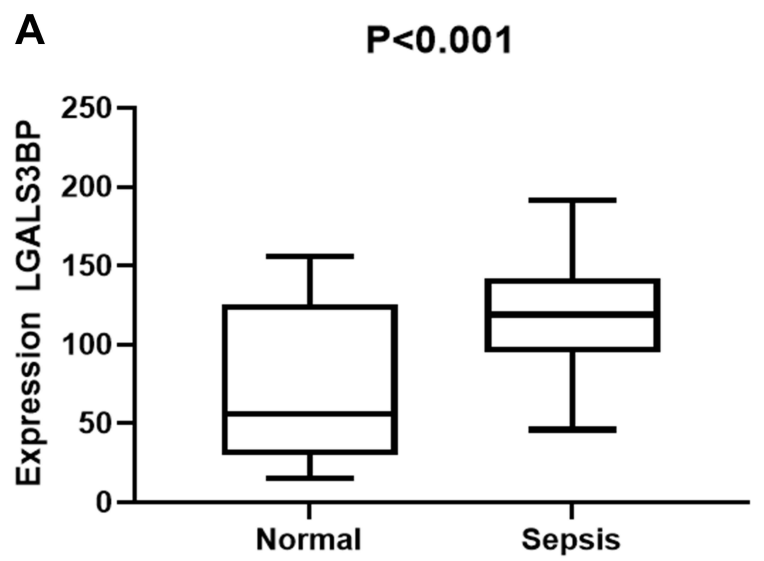

B $\quad P=0.002$

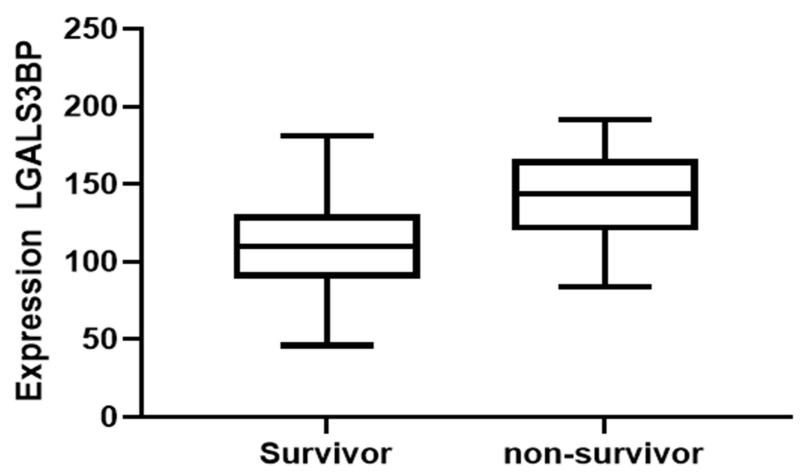

Figure 5 The ELISA results of LGALS3BP expression between normal and sepsis groups (A), survivors and non-survivors in the study (B). Normal control was $76.1 \pm 49.2$ $\mathrm{ng} / \mathrm{mL}$, sepsis group $119.8 \pm 32.7 \mathrm{ng} / \mathrm{mL}$, survivors $110.2 \pm 30.4 \mathrm{ng} / \mathrm{mL}$ and non-survivors $142.2 \pm 27.2 \mathrm{ng} / \mathrm{mL}$.
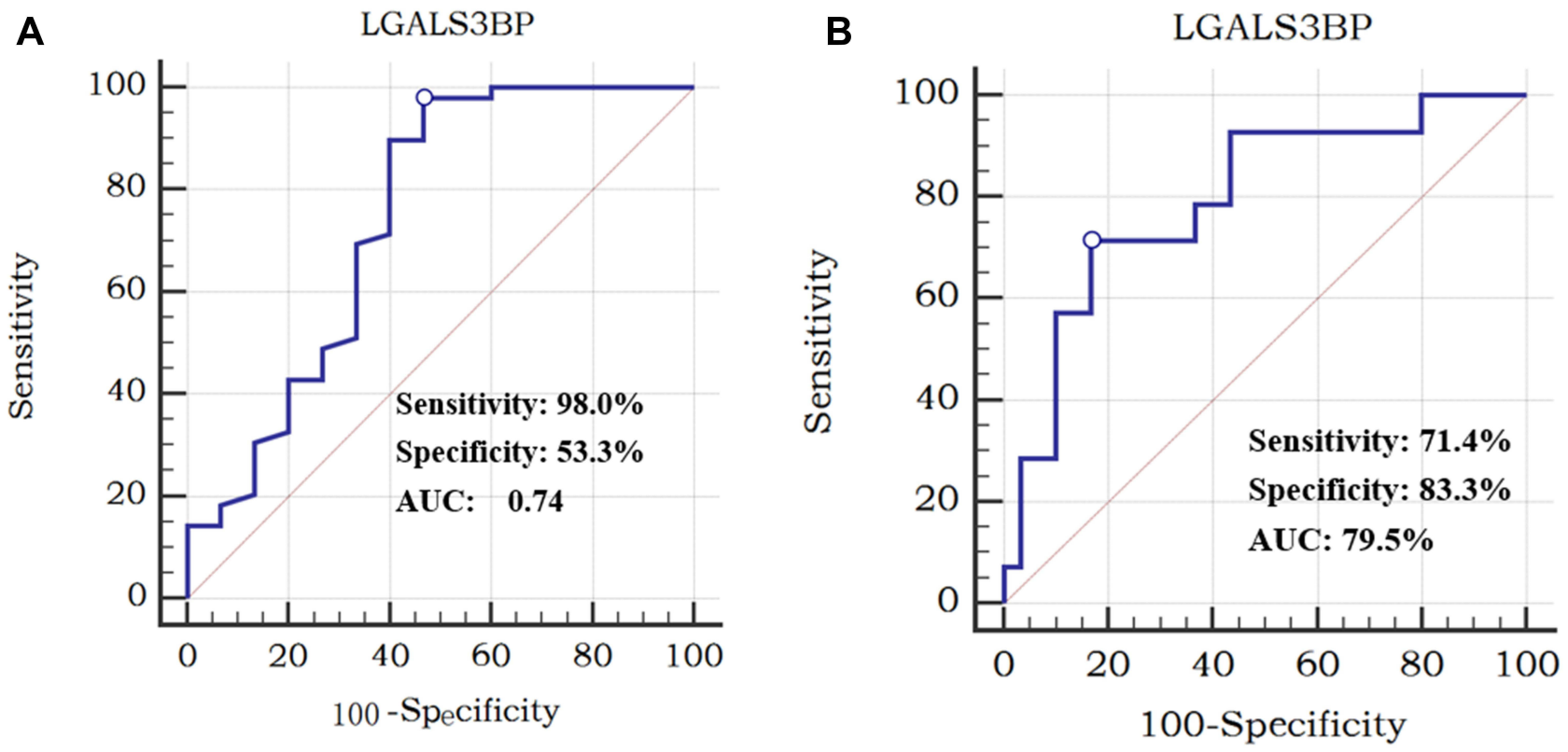

Figure 6 ROC curve of LGALS3BP for the comparison between non-sepsis vs sepsis patients (A); ROC curve analysis of LGALS3BP for the comparison of the comparison of the survivors vs non-survivors (B).

survivors and non-survivors, we found that it also has prognostic value in sepsis.

LGALS3BP, located on chromosome $17 \mathrm{q} 25$, is a ubiquitous and versatile secreted glycoprotein in the human body. It has a scavenger receptor cysteine-rich (SRCR) domain and is a member of the $\beta$-galactoside binding protein family. It is mainly involved in the regulation of the interaction between cells or between cells and the matrix. ${ }^{13}$ LGALS3BP can be synthesized and secreted by different types of cells, including haematopoietic cells and glandular or mucosal epithelial cells, and is present in human serum and other biological fluids (saliva, tears, breast milk and semen). ${ }^{14,15}$ This protein is related to the immune system, but its role and signal transduction mechanism in the host defence and immune response are still unknown.

At present, studies of LGALS3BP have focused on its role in tumours. Since LGALS3BP was discovered, it has been believed to be related to disease diagnosis, prognosis and the treatment response. Because it exists in the blood and is easy to detect, LGALS3BP has the potential to become a biomarker for malignant tumour diagnosis and 
prognosis prediction. LGALS3BP mainly affects the occurrence and development of malignant tumours through mechanisms such as cell adhesion, cell proliferation and migration, angiogenesis, and the immune defence against tumours. LGALS3BP has a dual role in the occurrence and development of malignant tumours. This protein can not only promote the development of malignant tumours, but also stimulate the body's immune response to inhibit tumour growth. Some studies showed that the specific function of LGALS3BP was related to its binding proteins. When combined with different regulatory proteins, LGALS3BP performs completely different or even opposite functions. ${ }^{16-20}$

Other studies showed that the level of LGALS3BP in the serum was abnormally increased when patients were infected with viruses such as hepatitis virus or human immunodeficiency virus or had a malignant tumour. ${ }^{21,22}$ Hong found that LGALS3BP could inhibit NF- $\mathrm{kB}$ signal transduction by studying HEK293T and RAW264.7 cells. TAK1 is a key mediator of NF- $\mathrm{BB}$ activation in the cellular stress response. LGALS3BP can interact with TAK1 to promote its degradation, thereby inhibiting $\mathrm{NF}-\kappa \mathrm{B}$ signal transduction and inhibiting inflammation. $^{23}$ A recent study showed that LGALS3BP was able to induce the expression of IL-6 by stromal cells in a galectin-3-dependent manner and thereby participate in COVID-19 infection. ${ }^{24}$

Some limitations of the present investigation should be noted. First, this study was based on patients with sepsis, and to determine whether LGALS3BP is a viable biomarker for sepsis it will be necessary to eventually compare septic patients without this condition. Second, the sample size of this study was small, and it's our further studies to expand the samples and related multi-center research. Finally, the specific mechanism of LGALS3BP in sepsis is currently unclear and subsequent experiments are needed to confirm these finding.

\section{Conclusion}

In summary, by combining DIA and ELISA results we conclude that LGALS3BP is abnormally elevated in the serum of patients with sepsis, and its level in non-survivors is higher than that in survivors. LGALS3BP is related to the severity of sepsis and had certain significance for patient prognosis; therefore, it has potential application value as a diagnostic marker and prognostic indicator of sepsis.

\section{Ethics Approval and Consent to Participate}

The study protocol and informed consent were approved by the Clinical Experiment Ethics Committee of Affiliated Hospital of Southwest Medical University (ethics NO: ky2018029). The clinical trial registration NO. was ChiCTR1900021261. Written informed consents were obtained from all study participants.

\section{Funding}

This study was sponsored by the Health Commission of Sichuan Province (20PJ138), Sichuan Science and Technology Department Project (2019JDPT0003).

\section{Disclosure}

The authors report no conflicts of interest in this work.

\section{References}

1. Hawiger J, Veach RA, Zienkiewicz J. New paradigms in sepsis: from prevention to protection of failing microcirculation. J Thromb Haemost. 2015;13(10):1743-1756. doi:10.1111/jth.13061

2. Yuki K, Murakami N. Sepsis pathophysiology and anesthetic consideration. Cardiovasc Hematol Disord Drug Targets. 2015;15 (1):57-69. doi:10.2174/1871529X15666150108114810

3. Adhikari NK, Fowler RA, Bhagwanjee S, et al. Critical care and the global burden of critical illness in adults. Lancet. 2010;376 (9749):1339-1346. doi:10.1016/S0140-6736(10)60446-1

4. Galley HF. Oxidative stress and mitochondrial dysfunction in sepsis. Br J Anaesth. 2011;107(1):57-64. doi:10.1093/bja/aer093

5. Rhodes A, Evans LE, Alhazzani W, et al. Surviving sepsis campaign: international guidelines for management of sepsis and septic shock: 2016. Intensive Care Med. 2017;43(3):304-377.

6. Meyer JG, Schilling B. Clinical applications of quantitative proteomics using targeted and untargeted data-independent acquisition techniques. Expert Rev Proteomics. 2017;14(5):419-429. doi:10.1080/14789450.2017.1322904

7. Tong $\mathrm{Y}, \mathrm{Ku} \mathrm{X}, \mathrm{Wu} \mathrm{C}$, et al. Data-independent acquisition-based quantitative proteomic analysis reveals differences in host immune response of peripheral blood mononuclear cells to sepsis. Scand J Immunol. 2019;89(4):e12748. doi:10.1111/sji.12748

8. Kim YM, Oh SW, Jeong SY, et al. Development of an ultrarapid one-step fluorescence immunochromatographic assay system for the quantification of microcystins. Environ Sci Technol. 2003;37 (9):1899-1904. doi:10.1021/es026191i

9. Ge SX, Son EW, Yao R. iDEP: an integrated web application for differential expression and pathway analysis of RNA-Seq data. $B M C$ Bioinform. 2018;19(1):534. doi:10.1186/s12859-018-2486-6

10. Szklarczyk D, Morris JH, Cook H, et al. The STRING database in 2017: quality-controlled protein-protein association networks, made broadly accessible. Nucleic Acids Res. 2017;45(D1):D362-D368. doi:10.1093/nar/gkw937

11. Shannon P, Markiel A, Ozier O, et al. Cytoscape: a software environment for integrated models of biomolecular interaction networks. Genome Res. 2003;13(11):2498-2504. doi:10.1101/gr.1239303

12. Su M, Guo C, Liu M, et al. Therapeutic targets of vitamin C on liver injury and associated biological mechanisms: a study of network pharmacology. Int Immunopharmacol. 2019;66:383-387. doi:10.1016/j.intimp.2018.11.048 
13. Läubli H, Alisson-Silva F, Stanczak MA, et al. Lectin galactoside-binding soluble 3 binding protein (LGALS3BP) is a tumor-associated immunomodulatory ligand for CD33-related Siglecs. J Biol Chem. 2014;289 (48):33481-33491. doi:10.1074/jbc.M114.593129

14. Koths K, Taylor E, Halenbeck R, et al. Cloning and characterization of a human Mac-2-binding protein, a new member of the superfamily defined by the macrophage scavenger receptor cysteine-rich domain. $J$ Biol Chem. 1993;268(19):14245-14249. doi:10.1016/S00219258(19)85233-X

15. Ullrich A, Sures I, D'Egidio M, et al. The secreted tumor-associated antigen $90 \mathrm{~K}$ is a potent immune stimulator. $J$ Biol Chem. 1994;269 (28):18401-18407. doi:10.1016/S0021-9258(17)32322-0

16. Correale M, Giannuzzi V, Iacovazzi PA, et al. Serum 90K/MAC-2BP glycoprotein levels in hepatocellular carcinoma and cirrhosis. Anticancer Res. 1999;19(4C):3469-3472.

17. Künzli BM, Berberat PO, Zhu ZW, et al. Influences of the lysosomal associated membrane proteins (Lamp-1, Lamp-2) and Mac-2 binding protein (Mac-2-BP) on the prognosis of pancreatic carcinoma. Cancer. 2002;94(1):228-239. doi:10.1002/cncr.10162

18. Qu H, Chen Y, Cao G, et al. Identification and validation of differentially expressed proteins in epithelial ovarian cancers using quantitative proteomics. Oncotarget. 2016;7(50):83187-83199. doi:10.18632/oncotarget.13077
19. Park SY, Yoon S, Kim H, et al. 90K glycoprotein promotes degradation of mutant $\beta$-catenin lacking the ISGylation or phosphorylation sites in the N-terminus. Neoplasia. 2016;18(10):618-625. doi:10.1016/j.neo.2016.08.006

20. Piccolo E, Tinari N, D'Addario D, et al. Prognostic relevance of LGALS3BP in human colorectal carcinoma. J Transl Med. 2015;13:248. doi:10.1186/s12967-015-0606-x

21. Iacobelli S, Natoli C, D'Egidio M, et al. Lipoprotein $90 \mathrm{~K}$ in human immunodeficiency virus-infected patients: a further serologic marker of progression. J Infect Dis. 1991;164(4):819. doi:10.1093/infdis/ 164.4.819

22. Artini M, Natoli C, Tinari N, et al. Elevated serum levels of $90 \mathrm{~K} /$ MAC-2 BP predict unresponsiveness to alpha-interferon therapy in chronic HCV hepatitis patients. J Hepatol. 1996;25(2):212-217. doi:10.1016/S0168-8278(96)80076-6

23. Hong CS, Park MR, Sun EG, et al. Gal-3BP negatively regulates NF$\kappa \mathrm{B}$ signaling by inhibiting the activation of TAK1. Front Immunol. 2019;10:1760. doi:10.3389/fimmu.2019.01760

24. Messner CB, Demichev V, Wendisch D, et al. Ultra-high-throughput clinical proteomics reveals classifiers of COVID-19 infection. Cell Syst. 2020;11(1):11-24. doi:10.1016/j.cels.2020.05.012
Infection and Drug Resistance

\section{Publish your work in this journal}

Infection and Drug Resistance is an international, peer-reviewed openaccess journal that focuses on the optimal treatment of infection (bacterial, fungal and viral) and the development and institution of preventive strategies to minimize the development and spread of resistance. The journal is specifically concerned with the epidemiology of
Dovepress

antibiotic resistance and the mechanisms of resistance development and diffusion in both hospitals and the community. The manuscript management system is completely online and includes a very quick and fair peerreview system, which is all easy to use. Visit http://www.dovepress.com/ testimonials.php to read real quotes from published authors. 